ARTIGO ORIGINAL

\title{
Atividade física e deficiência física: preferências, motivações e barreiras para a prática de atividade física
}

\section{Physical activity and physical disability: preferences, motivations and barriers for the practice of physical activity}

Luciana Erina Palma, Bhianca Conterato Patias, Roberta Marostega Feck

Universidade Federal de Santa Maria (UFSM), Santa Maria/RS, Brasil

\section{HISTÓRICO DO ARTIGO}

Recebido: 04 maio 2020

Revisado: 06 julho 2020

Aprovado: 21 julho 2020

\section{PALAVRAS-CHAVE:}

Deficiência Física; Motivação; Barreiras; Preferências.

\section{KEYWORDS:}

Physical disability; Motivation; Barrier; Preferences.

\section{RESUMO}

OBJETIVO: Objetivou-se identificar as preferências, barreiras e motivações para a prática de atividade física por pessoas com deficiência física.

MÉTODOS: A pesquisa é de caráter qualitativo, do tipo descritivo. Participaram do estudo 34 indivíduos com deficiência física, 27 eram ativos e 7 inativos, com média de idades de 35,5 anos. Para as coletas dos dados, utilizaram-se questionários sobre preferência, motivações e barreiras para prática de atividade física.

RESULTADOS: A partir dos resultados, as preferências identificadas envolvem a prática esportiva, especialmente, o basquetebol em cadeira de rodas. Sobre as motivações, a dimensão saúde se sobressaiu como o fator motivacional mais citado. Quanto às barreiras, a falta de equipamentos disponíveis e adaptados foi a mais evidenciada para a prática de atividades físicas.

CONCLUSÃO: Estes resultados subsidiam a criação de atividades específicas considerando as preferências e as motivações apresentadas pelas pessoas com deficiência física, aliado à tentativa de minimizar as barreiras para esta prática.

\section{ABSTRACT}

OBJECTIVE: The objective was to identify preferences, barriers and motivations for the practice of physical activity by people with physical disabilities.

METHODS: The research is qualitative, of the descriptive type. Thirty-four individuals with physical disability participated in the study, 27 were active and 7 were inactive, with an average age of 35.5 years. For data collection, questionnaires were used on preferences, motivations and barriers for the practice of physical activity.

RESULTS: From the results, the identified preferences involve the sports practice, mainly, the wheelchair basketball. On the motivations, the health dimension stood out as the most cited motivational factor, both by the active and the inactive subjects. As for the barriers, the lack of equipment available and adapted was the most evidenced for the practice of physical activities.

CONCLUSION: These results subsidize the creation of specific activities considering the preferences and motivations presented by people with physical disabilities, along with the attempt to minimize the barriers for this practice. 


\section{INTRODUÇÃO}

No final do século XIX e início do XX começaram os primeiros estudos sobre atividade física relacionada à saúde, voltada especificamente para tratamento de doenças e recuperações de lesões. A atividade física é caracterizada como sendo qualquer movimento corporal voluntário capaz de produzir um gasto energético acima dos níveis de repouso (NAHAS; GARCIA, 2010).

Com base no Censo do IBGE (2010), no Brasil 23,9\% da população apresenta algum tipo de deficiência. Conforme a Lei $n^{\circ}$. 13.146/2015, no seu Artigo 112, inciso III, deficiência é definida como:

[...] aquela que tem impedimento de longo prazo de natureza física, mental, intelectual ou sensorial, o qual, em interação com uma ou mais barreiras, pode obstruir sua participação plena e efetiva na sociedade em igualdade de condições com as demais pessoas.

Mais especificadamente, a deficiência física refere-se ao comprometimento do aparelho locomotor, abrangendo assim os sistemas osteoarticular, muscular e o nervoso. Quaisquer destes sistemas sendo afetados por doenças ou lesões podem causar quadros de limitações físicas, tendo grau e gravidade variáveis (FRONTERA et al. apud FENATO, 2007).

Ao abordar a atividade física para as pessoas com deficiência física, percebe-se que há uma redução tanto no número de estudos, bem como no número de participação destas e pessoas em atividades físicas (GREGUOL, 2017). No entanto, sabe-se dos benefícios da prática de atividades físicas e esportivas para a manutenção e melhora tanto da saúde quanto da aptidão física (WHO, 2016).

Ratificando, para Nahas (2006, p. 139), "as atividades físicas regulares podem reduzir os sintomas de ansiedade e depressão, promover a socialização e aumentar os níveis e bem-estar geral das pessoas com deficiência". Melo e Lopes (2002) relatam que a prática de atividade física por pessoas com deficiência é uma oportunidade de testar seus limites e potencialidades, prevenir as enfermidades secundárias a sua deficiência e promover a integração social. Também, a prática esportiva influencia na melhora da aptidão física, ganho de independência, socialização, autoconfiança e autoestima para esta população (ARAÚJO, 2011).

Diante dos benefícios, os motivos pelos quais as pessoas com deficiência buscam e permanecem na prática de atividade física são os mais diversos (melhora da saúde, lazer, condicionamento, etc.), em que reconhecê-los é algo fundamental para auxiliar na criação de atividades específicas e melhor direcioná-las a este público (SAITO, 2007). Para Samulski (2002), a motivação é caracterizada como um processo ativo, intencional e conduzido a um objetivo, dependendo da interação de fatores pessoais (intrínsecos) e fatores ambientais (extrínsecos). Chiavenato (2010) afirma que a motivação está intimamente relacionada com o comportamento e desempenho das pessoas, também se sobressai como um importante elemento na compreensão do comportamento humano em suas particularidades.

Neste contexto, emergem as preferências de cada pessoa por determinadas práticas, sendo estas influenciadas por diversos fatores (FERRAZ, 2005). O referido autor ainda salienta que as preferências são frequentemente determinadas pela personalidade, pela influência exercida pelo meio cultural ao qual a pessoa está inserida e que algumas atividades têm sua própria identidade, relacionadas ao movimento empregado, e isto pode ser um limitador ou facilitador para a opção de sua prática.

Também, relacionadas à prática de atividade física, percebe-se a existência de diversas barreiras que dificultam ou impedem esta prática. Para Brows (2005), as barreiras são entendidas como obstáculos observados pelo sujeito que podem reduzir o engajamento em comportamentos saudáveis. Pela Lei Brasileira de Inclusão da Pessoa com Deficiência (BRASIL, 2015), as barreiras são definidas como:

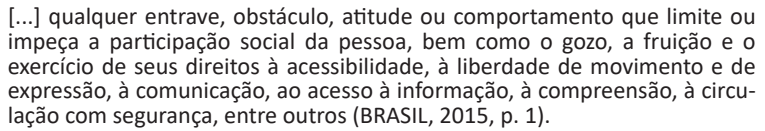

Identificar as barreiras para a participação em alguma prática de atividade física parece ser uma estratégia e assim, a partir disso, estabelecer novos hábitos e redução dos níveis de sedentarismo, importante para toda e qualquer população (CESCHINI; FIGUEIRA JÚNIOR, 2007). Nessa mesma perspectiva, Seron, Arruda e Greguol (2015) concluíram seu estudo reforçando a necessidade de levantar dados com relação à inclusão de pessoas com deficiência em programas de atividades físicas, para assim elencar e assinalar fatores que possam vir a contribuir na construção de programas e políticas públicas locais, diminuindo o sedentarismo e automaticamente aumentando a qualidade de vida entre essa população.

Ainda Vasudevan, Rimmer e Kviz apud Greguol (2017, p. 12) afirmam que a "disseminação do conhecimento sobre os benefícios e as possibilidades de prática de atividades físicas e esportivas por essa população é peça-chave para a melhor adesão aos programas disponíveis". Assim, associado à disseminação, aos benefícios e ao envolvimento ou não dos indivíduos com deficiência em alguma prática de atividade física, este estudo teve como objetivo identificar as motivações, as preferências e as barreiras para a prática de atividade física por pessoas com deficiência física

\section{MÉTODOS}

A pesquisa se desenvolveu sob caráter qualitativo (THOMAS; NELSON; SILVERMAN, 2012) do tipo descritivo que, segundo Gil (2010), tem como principal objetivo descrever características de determinada população ou fenômeno e, para Sampieri (2006), os estudos descritivos se centram em coletar dados que mostrem um evento, uma comunidade, um fenômeno, feito, contexto ou situação que ocorre.

A escolha do público envolvido foi por conveniência (GIL, 2010). Desse modo, 34 indivíduos com deficiência física foram avaliados pelo estudo, destes, 27 eram ativos e 7 eram inativos, apresentando média de idade de 35,5 anos. Com relação aos ativos, os mesmos estavam inseridos na prática de atividades físicas a mais de um ano, e os inativos desde a aquisição da deficiência não praticavam nenhum tipo de atividade. Ressalta-se que o estudo não procurou investigar esta população anteriormente a deficiência. Possuir deficiência intelectual associada ou não participar na totalidade do estudo foram considerados critérios para exclusão.

A coleta dos dados foi realizada com equipes de Basquete- 
bol em Cadeiras de Rodas (BCR) que participavam de uma competição da modalidade entre equipes, em uma cidade do Rio Grande do Sul (RS), os quais compõem a amostra dos indivíduos ativos, e os expectadores desta competição compõem a amostra dos indivíduos inativos. Foi aproveitada a oportunidade para a realização da pesquisa, pois ao mesmo tempo em que reuniu um grupo de pessoas que se enquadrariam nos critérios de inclusão da pesquisa, também contemplou com pessoas de quase todos os municípios do estado já referido.

Os instrumentos foram aplicados no dia da competição com os indivíduos, quando estes estavam no intervalo dos jogos. Estabeleceu-se uma conversa inicial realizada de maneira individual, em que foram explicados os objetivos da pesquisa, logo após foi feita a leitura e assinatura do Termo de Consentimento Livre e Esclarecido (TCLE), assinado em duas vias. Caso o indivíduo com deficiência física apresentasse limitações motoras nos membros superiores, por quaisquer motivos, o pesquisador realizou a leitura e preenchimento dos instrumentos, obtendo as respostas do participante oralmente.

Para o estudo, foi utilizada uma ficha de identificação baseado em Gil (2010) contendo informações acerca dos indivíduos participantes, caracterizando-os. Para identificar as preferências para a prática de atividade física, serviu de base para elaboração dos questionários as orientações de Thomas, Nelson e Silverman (2012), que apresenta os principais itens que devem compor um questionário. Considerando essas orientações de base dos autores supracitados elaborou-se o questionário contendo questões que indicavam as preferências por determinado tipo de atividade física ou qual gostaria de praticar, bem como o tempo de prática e o porquê da escolha de determinada atividade.

Para avaliar a motivação, foi utilizado um questionário construído e validado por Balbinotti e Barbosa (2006) denominado Inventário Motivacional á Prática Regular de Atividade FísicaIMPRAF-54. O instrumento avalia seis dimensões associadas à motivação para a realização de atividades físicas regulares, sendo elas: Controle de Estresse (CE), Saúde (Sa), Sociabilidade (So), Competitividade (Co), Estética (Es) e Prazer (Pr). As respostas são dadas de acordo com uma escala do tipo Likert, bidirecional graduada de 1 a 5 pontos, distribuídos da seguinte forma: 1 - Isto me motiva pouquíssimo; 2 - Isto me motiva pouco; 3 Mais ou menos - não sei dizer -tenho dúvida; 4 - Isto me motiva muito; 5 - Isto me motiva muitíssimo.

Para analisar as barreiras, utilizou-se um questionário adaptado para pessoas com deficiência física elaborado por Martins e Petroski (2000), que avalia a percepção de barreiras para a prática de atividade física. Conforme o instrumento, para uma barreira ser considerada importante, é necessário que seja indicada como "quase sempre" ou "sempre". Sobre o questionário foram considerados os fatores que indicavam com que frequência eles representam motivos para não praticar atividades físicas. Citamos alguns itens acrescentados ao questionário, que são fundamentais para a população específica do estudo como: a falta de acompanhante, dificuldade de locomoção independente, falta de equipamentos adaptados, falta de opção de prática, falta de transporte acessível, falta de acessibilidade nos espaços de prática, falta de profissionais capacitados.

A análise dos dados deu-se de maneira descritiva, primeiramente foram separadas as respostas dos inativos e dos ativos, após foram agrupadas as respostas em preferências, motivação e barreiras para a prática de atividade física, e por fim assinalan- do todas as variáveis envolvidas. O estudo se encontra aprovado sob Parecer $N^{\circ}$. 1.101.807 e registrado pelo Comitê de Ética em Pesquisa da Universidade Federal de Santa Maria (UFSM), sob o número CAAE: 42822515.1.0000.5346.

\section{RESULTADOS}

Os dados obtidos no estudo foram sistematizados na forma de quadros, seguidos de suas discussões. Os participantes são divididos entre Ativos (A) e Inativos (I). Na tabela, a letra "A" indica que o indivíduo é Ativo, e a letra "I" que ele é Inativo.

Num primeiro momento é apresentada a identificação dos participantes (Quadro 1 e 2), indicando a deficiência de cada um e, no caso dos ativos, também a atual prática de atividade física. A seguir apresentamos os dados sobre as preferências, motivação e barreiras (Quadros 3, 4 e 5). Essa ordem foi escolhida por entender que apresenta uma sequência lógica, primeiro, identificar quais as atividades físicas esses indivíduos têm como preferência, em seguida, o que os motiva a iniciarem uma atividade física ou continuarem em alguma, e por último, as barreiras mostrando o que se torna um empecilho para que esses indivíduos possam realizar as atividades físicas que desejam.

Quadro 1. Identificação dos indivíduos participantes ativos e suas deficiências.

\begin{tabular}{|c|c|c|}
\hline Participantes & Atual Prática & Deficiência \\
\hline $\begin{array}{l}\text { A1, A2, A10, } \\
\text { A14,A18, } \\
\text { A26 }\end{array}$ & Basquetebol em Cadeira de Rodas & Lesão Medular \\
\hline $\mathrm{A} 11, \mathrm{~A} 13$ & Basquetebol em Cadeira de Rodas & Amputação \\
\hline A15 & Basquetebol em Cadeira de Rodas & Mielomeningocele \\
\hline A24 & Basquetebol em Cadeira de Rodas & Sequelas de Poliomelite \\
\hline A27 & Basquetebol em Cadeira de Rodas & Hidrocefalia \\
\hline A3 & Basquetebol em Cadeira de Rodas & $\begin{array}{l}\text { Traumatismo Raqui-Me- } \\
\text { dular }\end{array}$ \\
\hline A7 & Basquetebol em Cadeira de Rodas & Má Formação Congênita \\
\hline A8 & Basquetebol em Cadeira de Rodas & Deficiência Motora \\
\hline A20 & $\begin{array}{l}\text { Basquetebol em Cadeira de Rodas, } \\
\text { Atletismo }\end{array}$ & Deficiência Motora \\
\hline A9 & $\begin{array}{l}\text { Basquetebol em Cadeira de Rodas, } \\
\text { caminhada }\end{array}$ & Sequelas de Poliomelite \\
\hline A23 & $\begin{array}{l}\text { Basquetebol em Cadeira de Rodas, } \\
\text { Canoagem, Corrida de Rua }\end{array}$ & Lesão Medular \\
\hline A5 & $\begin{array}{l}\text { Basquetebol em Cadeira de Rodas, } \\
\text { Futebol }\end{array}$ & Sequelas de Poliomelite \\
\hline A17 & $\begin{array}{l}\text { Basquetebol em Cadeira de Rodas, } \\
\text { Halterofilismo, Musculação }\end{array}$ & Lesão Medular \\
\hline A22 & $\begin{array}{l}\text { Basquetebol em Cadeira de Rodas, } \\
\text { Handbike }\end{array}$ & Mielomeningocele \\
\hline A19 & $\begin{array}{l}\text { Basquetebol em Cadeira de Rodas, } \\
\text { Handbike, Canoagem, Halterofilismo, } \\
\text { Maratona }\end{array}$ & Lesão Medular \\
\hline A25 & $\begin{array}{l}\text { Basquetebol em Cadeira de Rodas, } \\
\text { Handebol em Cadeira de Rodas }\end{array}$ & Sequelas de Poliomelite \\
\hline A16 & $\begin{array}{l}\text { Basquetebol em Cadeira de Rodas, } \\
\text { Musculação }\end{array}$ & Mielomeningocele \\
\hline A4 & $\begin{array}{l}\text { Basquetebol em Cadeira de Rodas, } \\
\text { Natação, Handbike }\end{array}$ & Lesão Medular \\
\hline A6 & Musculação & Amputação \\
\hline A12, A21 & Musculação & Lesão Medular \\
\hline
\end{tabular}

Fonte: Autoras.

Quadro 2. Identificação dos indivíduos participantes inativos e sua 
deficiência.

\begin{tabular}{|ll|}
\hline Participantes & Deficiência \\
\hline $11,12,13,14,17$ & Amputação \\
\hline 15 & Lesão Medular \\
\hline 16 & Atrofia Muscular Progressiva \\
\hline
\end{tabular}

Fonte: Autoras.

Dados obtidos na ficha de identificação do estudo, caracterizando os participantes indicam que, entre os ativos, a maioria é acometida pela lesão medular, de ordem traumática. Como atual prática de atividade física, destaca-se o basquetebol em cadeira de rodas (BCR), sendo isto uma variável influenciada pelo contexto em que a pesquisa foi realizada. Os participantes inativos, os quais não praticam atividades físicas, em sua maioria são amputados e apresentavam média de idade de 35,5 anos, sendo todos do sexo masculino. No Quadro 3, se apresentam as preferências de prática de atividade física relatadas pelos participantes ativos e inativos.

Quadro 3. Preferências para prática de atividade física pelos indivíduos ativos.

\begin{tabular}{|c|c|}
\hline Participantes & Preferência \\
\hline \multicolumn{2}{|l|}{ Ativos } \\
\hline $\mathrm{A} 2$ & Automobilismo \\
\hline $\begin{array}{l}A 1, A 3, A 4, A 8, A 10, A 15 \\
A 19, A 20, A 21, A 22, A 26\end{array}$ & Basquetebol em Cadeira de Rodas \\
\hline A5 & Tênis, Surf \\
\hline $\mathrm{A} 6, \mathrm{~A} 16, \mathrm{~A} 25$ & Natação \\
\hline $\mathrm{A} 7, \mathrm{~A} 27$ & Handebol em Cadeira de Rodas, \\
\hline A9, A12 & Musculação \\
\hline A11 & Artes Marciais \\
\hline A13 & $\begin{array}{l}\text { Handebol em Cadeira de Rodas, Lançamento } \\
\text { de Dardo e Arremesso de Disco. }\end{array}$ \\
\hline A14 & Remo \\
\hline A17, A24 & Canoagem \\
\hline A18 & Tênis, Canoagem, Bocha e Dança \\
\hline A23 & $\begin{array}{l}\text { Basquetebol em Cadeira de Rodas, Canoagem, } \\
\text { Corrida de Rua }\end{array}$ \\
\hline \multicolumn{2}{|l|}{ Inativos } \\
\hline 11 & Caça \\
\hline 12,13 & Caminhada \\
\hline 14 & Natação, Basquete \\
\hline 15 & $\begin{array}{l}\text { Basquetebol, Futebol, Dança, Handbike, Bocha, } \\
\text { Natação, Tiro ao Alvo }\end{array}$ \\
\hline 16 & Hipismo \\
\hline 17 & Não respondeu \\
\hline
\end{tabular}

Fonte: Autoras.

Com relação aos ativos, a maioria relatou como preferência a prática do $\mathrm{BCR}$, sendo esta a atual prática deles. Destacamos que este é um ponto frágil no estudo, necessitando de outras pesquisas em outros contextos de participação, pois esses dados com relação às preferências podem ter sido influenciados pelo ambiente pesquisado, sendo ele uma competição que envolvia a modalidade em questão. Porém, neste estudo sugere-se que os participantes se sintam satisfeitos com a modalidade praticada e que atenda os objetivos e as demandas destes indivíduos, sejam elas de reabilitação, inclusão social ou prática em nível de competição.

Outras preferências foram colocadas pelos indivíduos, como por exemplo, o Handebol em cadeira de rodas, natação, canoagem, tênis, bocha, remo, artes marciais, atletismo (dardo, disco), surf, automobilismo, musculação e dança. Estudo semeIhante, de Santos e Knijnik (2006, p. 29), apontam também uma variedade de preferências que se aproxima com os resultados obtidos neste estudo, como a musculação, caminhada, treino aeróbio por meio de esteira e bicicleta ergométrica; ginástica; natação; alongamento; corrida, handebol adaptado, pilates, tênis, dentre outros. Conforme Lovisolo (1997), a preferência é algo fortemente individual, ao mesmo tempo em que é amplamente coletivizado, assim o gosto ou a preferência não têm somente a função da identidade pessoal individual, mas também a construção de identidades coletivas, de grupos. De maneira geral, há um direcionamento maior das preferências para os esportes. Nesta direção, Ferraz (2005) nos diz que o esporte é uma prática frequente, não só pelo seu caráter competitivo, mas por fatores que ultrapassam as demandas físicas e fisiológicas, ou seja, vai além do simples ato de praticar. Inferem-se, também, as possibilidades oferecidas no esporte, tanto em nível de participação quanto em nível competitivo.

Em relação aos inativos, as preferências citadas abrangem a caminhada, caça, hipismo, natação e basquetebol em cadeira de rodas. As opções elencadas são bem restritas perante as várias possibilidades de prática que esta população poderia estar inserida, isto pode ser explicado pela falta de conhecimento das suas possibilidades e das diversas modalidades, ou da deficiência ainda estar sendo percebida pelo indivíduo como o limitador. Para Seronet et al. (2015, p. 18), "o fato de muitos sujeitos relatarem que as limitações físicas os impedem de ser fisicamente ativos pode refletir a falta de informação sobre suas reais possibilidades de prática".

Apenas o indivíduo 15 se destaca quando cita várias práticas a qual prefere ou gostaria de estar inserido. Pode-se dizer que isto está relacionado às experiências já vivenciadas e ao conhecimento das possibilidades de prática de atividades físicas. Neste cenário, Ferraz (2005) afirma que a escolha/preferência pela prática de atividade física é determinada por fatores diferenciados, a própria maneira como se realizam determinadas atividades físicas é um fator que irá facilitar ou dificultar a adesão à prática. A seguir, apresentamos as motivações para a prática de atividade física tanto para os indivíduos ativos, quanto para os inativos (Quadro 4).

Quadro 4. Motivação para a prática de atividade física pelos indivíduos ativos.

\begin{tabular}{|cl|}
\hline Fatores Motivantes & Fatores Menos Motivantes \\
\hline Ativos & \\
\hline Saúde & Estética \\
\hline Prazer & Competitividade \\
\hline Sociabilidade & Controle de estresse \\
\hline Inativos & \\
\hline Saúde & Competitividade \\
\hline Prazer & Estética \\
\hline Sociabilidade & Controle de estresse \\
\hline
\end{tabular}

Fonte: Autoras. 
Alguns estudos (PAGANI, 2012; TORRALBA; VIEIRA; RUBIO, 2017) vem demonstrando que a motivação quiçá seja o fator mais importante que atletas permaneçam e continuem numa determinada modalidade, também determinada com um dos fatores psicológicos de grande relevância para o sucesso desportivo. Nessa direção, segundo alguns autores, a atmosfera motivacional pode ter impacto na regulação da motivação dos atletas e também pode facilitar ou impedir a satisfação das suas necessidades psicológicas básicas (SARRAZIN; BOICHÉ; PELLETIER, 2007).

Baseados em Souza et al. (2017, p. 106) entendemos que a motivação "é uma ferramenta que estimula o indivíduo a se manter em uma determinada atividade física e pode influenciar a permanência e a intensidade do praticante". Ainda, de uma forma mais ampla, para Cratty (1983), significa os fatores que levam as pessoas a uma ação em diferentes situações. Assim, o estudo dos motivos implica em verificar as razões pelas quais se escolhe fazer algo ou executar algumas tarefas com maior empenho do que outras ou, ainda, permanecer numa atividade por um período de tempo maior.

Conforme estudo desenvolvido por Palma et al. (2016) relacionado a tendências competitivas de atletas de basquetebol em cadeira de rodas verificou-se que a tendência predominante foi em competir (TC), apontando que os indivíduos gostam de competir pelo prazer inerente ao esporte, sem haver preocupação em ganhar. Ou seja, a competitividade também não se destaca neste estudo, não sendo um fator de grande importância motivacional.

Também, em estudo recente publicado por Silva et al. (2019), relacionado a orientação motivacional de atletas de Basquetebol em Cadeiras de Rodas (BCR), Rugby em Cadeiras de Rodas (RBC) e Handebol em Cadeira de Rodas (HCR) em situação de competição, de maneira geral, os resultados apontaram que os atletas apresentaram maior escore na tendência a motivação orientada para a tarefa e não ao ego, ou seja, há uma preocupação na melhoria das suas próprias capacidades e não em querer superar ou ser superior os outros.

Neste estudo, percebe-se que tanto os indivíduos ativos, quanto os inativos, citaram como principal fator motivante para a prática de atividade física a saúde, seguido de prazer e sociabilidade. Corroborando com estudo realizado por Seron, Arruda e Greguol (2015) com pessoas com deficiência motora, apontando que "quando perguntados sobre os três principais fatores que os levaram a iniciar a prática de atividade física, $72 \%$ consideraram o fator "porque é bom para a saúde". Nota-se a preocupação dos indivíduos com deficiência em relação a este fator, sendo possível indicar que a prática de atividade física pode se estabelecer como um meio para minimizar os danos e potencializar as capacidades dos indivíduos. Reafirmando, em estudo realizado por Palma et al. (2017), objetivando identificar a motivação para a prática de atividade física direcionadas a pessoas com deficiência visual, também mostrou que há uma preocupação significativa com a saúde, evidenciando assim a importância deste fator mesmo abrangendo outra deficiência.

Um estudo semelhante, realizado por Cardoso, Palma e Zanella (2010), objetivando identificar a motivação para a prática do esporte adaptado por pessoas com deficiência, teve como resultados que as principais dimensões da motivação para a prática de atividades físicas são a sociabilidade, o prazer e a saúde, sendo que estas dimensões nos permitem considerá-las relevantes para a qualidade de vida desta população. Neste estudo, estas dimensões também foram apontadas como fatores motivadores para a prática de atividade física por pessoas com deficiência física.

Quanto aos fatores menos motivantes, entre os indivíduos ativos, é citado à estética, seguido de competitividade e controle de estresse. Entre os inativos, o fator menos motivador é a competitividade, posteriormente à estética e, por fim, o controle de estresse. Os resultados indicam que estas dimensões não são fatores considerados motivantes para a prática de atividade física por pessoas com deficiência física participantes deste estudo.

Cabe destacar que as motivações apresentadas pelos participantes não são necessariamente permanentes ao longo da vida, podendo ser modificadas conforme as conjunturas vividas por cada indivíduo. Para Machado (1997), alguns motivos podem adquirir maior importância sobre outros em determinadas circunstâncias, direcionando os indivíduos para diversos objetivos. Assim como, diferentes motivos podem estimular a diferentes indivíduos a realizarem a mesma atividade.

Na sequência são apresentados os resultados encontrados referentes às barreiras para a prática de atividade física indicadas pelos participantes, separando-os novamente entre os indivíduos ativos e os inativos (Quadro 5).

Quadro 5. Frequência das barreiras para a prática de atividade física pelos indivíduos ativos.

\begin{tabular}{|c|c|c|c|c|}
\hline & \multicolumn{4}{|c|}{ Frequência } \\
\hline & Sempre & Quase Sempre & Raramente & Nunca \\
\hline \multirow{2}{*}{ 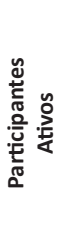 } & $\begin{array}{l}\text { Falta de } \\
\text { equipamentos } \\
\text { disponíveis e } \\
\text { adaptados; }\end{array}$ & $\begin{array}{l}\text { Jornada de } \\
\text { trabalho } \\
\text { extensa; }\end{array}$ & $\begin{array}{l}\text { Falta de } \\
\text { habilidades } \\
\text { físicas. }\end{array}$ & $\begin{array}{l}\text { Falta de interes- } \\
\text { se em praticar; }\end{array}$ \\
\hline & $\begin{array}{l}\text { Falta de trans- } \\
\text { porte acessível; }\end{array}$ & $\begin{array}{l}\text { Falta de } \\
\text { equipamentos } \\
\text { disponíveis e } \\
\text { adaptados; }\end{array}$ & & $\begin{array}{l}\text { Falta de incen- } \\
\text { tivo da família e } \\
\text { amigos. }\end{array}$ \\
\hline \multirow{2}{*}{ 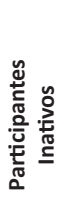 } & $\begin{array}{l}\text { Falta de } \\
\text { equipamentos } \\
\text { disponíveis e } \\
\text { adaptados; }\end{array}$ & $\begin{array}{l}\text { Falta de opção } \\
\text { de prática; }\end{array}$ & $\begin{array}{l}\text { Compromissos } \\
\text { familiares. }\end{array}$ & $\begin{array}{l}\text { Falta de incen- } \\
\text { tivo da família e } \\
\text { amigos; }\end{array}$ \\
\hline & $\begin{array}{l}\text { Medo de lesio- } \\
\text { nar-se. }\end{array}$ & $\begin{array}{l}\text { Falta de } \\
\text { companhia. }\end{array}$ & & $\begin{array}{l}\text { Preocupação } \\
\text { com a aparên- } \\
\text { cia. }\end{array}$ \\
\hline
\end{tabular}

Fonte: Autoras.

A falta de equipamentos disponíveis e adaptados é a barreira mais evidenciada para a prática de atividades físicas por pessoas com deficiência física neste estudo, abarcando tanto os indivíduos ativos, quanto os inativos. Percebe-se assim que ainda há uma carência no atendimento à população com deficiência física em relação aos equipamentos adequados e disponíveis conforme as características e peculiaridades. Resultados esses também encontrados em estudo de Seron, Arruda e Greguol (2015), que apontam que $61 \%$ dos entrevistados indicaram como barreiras a falta de equipamentos específicos.

Outra barreira citada pelos indivíduos ativos é a falta de transporte acessível diferentemente de estudo realizado por 
Seron, Arruda e Greguol (2015, p. 219), em Londrina (PR), que aponta que a "falta de transporte e de acessibilidade arquitetônica não foram barreiras identificadas para os indivíduos do estudo". Já o que quase sempre se torna uma barreira entre os ativos é a jornada de trabalho dando ênfase à autonomia destes sujeitos.

Em consonância, em estudo realizado por Marmeleira et al. (2018), direcionado à deficiência visual, objetivando também avaliar as barreiras para a prática de atividade física, concluiu-se que as barreiras que mais fortemente impedem ou dificultam são a má conservação das calçadas, a falta de instalações apropriadas ou com condições de segurança, a falta de políticas públicas e programas comunitários específicos.

Quanto aos indivíduos inativos, além da falta de equipamentos disponíveis e adaptados, outra barreira frequentemente citada é o medo de lesionar-se. Também a falta de opção de prática e falta de companhia quase sempre se torna uma barreira, assim percebe-se o desconhecimento das possibilidades de prática e seus benefícios, bem como o fator de dependência de outras pessoas, podendo justificar-se pelo grau da deficiência ou pela acomodação do indivíduo junto à família.

Destaca-se neste estudo, que a falta de interesse pela prática e à falta de incentivo da família e amigos nunca é uma barreira para estar inserido em uma prática de atividade física entre os indivíduos ativos. E o que raramente afeta a prática pelos indivíduos ativos é a falta de habilidades físicas, pois sabem que são indivíduos capazes dentro da modalidade que praticam. Contrapondo estes achados, têm-se o estudo de Seron, Arruda e Greguol (2015) em que 67\% relataram falta de interesse pela prática, bem como $72 \%$ das pessoas investigadas pontuaram a falta de energia ou cansaço físico, falta de recursos financeiros e falta de habilidades físicas como fatores que atuam como barreiras para a prática de atividade física. Para os inativos, a falta de incentivo da família e amigos e a preocupação com a aparência não são mencionados como impedimentos para a prática de atividade física, evidenciando-se que há o incentivo para o início de uma prática de atividade física.

\section{CONCLUSÃO}

Os resultados deste estudo mostraram que as preferências de prática de atividade física apresentadas pelas pessoas com deficiência física ativas abrangem a prática esportiva, especialmente, o BCR. Assinala-se aqui uma dificuldade neste estudo, em que as preferências podem ter sido influenciadas pelo contexto de coleta de dados, sugerindo assim realizar novas pesquisas em outros ambientes, com novos participantes. Sobre as motivações para a prática de atividade física, a dimensão da saúde se sobressaiu como o fator motivacional mais citado, tanto pelos indivíduos ativos quanto os inativos. Da mesma forma, a falta de equipamentos disponíveis e adaptados foi à barreira mais evidenciada para a prática de atividades física por pessoas com deficiência física.

Ao se reconhecer as preferências, motivações e barreiras relacionadas à prática de atividade física por pessoas com deficiência física têm-se subsídios para a criação de programas diversificados que atendam as expectativas, interesses e demandas desta população. Aos profissionais, tornam-se elementos essenciais, agindo como facilitadores de uma ação futura. Por fim, ao identificar esses fatores direcionados a pessoas com deficiência, é possível difundir a prática de atividade física, bem como aumentar a inserção e estimular a permanência.

A partir disso, pode-se oferecer mais suporte para a atuação de profissionais de Educação Física, considerando que a prática de atividade física é um importante fator para a prevenção, recuperação e manutenção da qualidade de vida de toda e qualquer pessoa. Também se salienta que a atuação do profissional de Educação Física deve buscar a realização de atividades as quais sejam compatíveis e considerem as suas individualidades.

\section{REFERÊNCIAS}

ARAÚJO, P. F. Desporto adaptado no Brasil. São Paulo: Phorte, 2011.

BALBINOTTI, M. A. A.; BARBOSA, M. L. L. inventário de motivação à pratica regular de atividade física (IMPRAF-54). Laboratório de Psicologia do Esporte. Universidade Federal do Rio Grande do Sul: Porto Alegre, 2006.

BRASIL. Lei no 13.146, de 6 de julho de 2015. Institui a Lei Brasileira de Inclusão da Pessoa com Deficiência (Estatuto da Pessoa com Deficiência). Diário Oficial da União, 2015. Disponível em: <http://www.planalto.gov.br/ ccivil 03/ ato2015-2018/2015/lei//13146.htm>. Acessado em: 28 de julho de 2019.

BROWN, S. A. Measuring perceived benefits and perceived barriers for physical activity. American Journal of Health Behavior, Oak Ridge, v. 29, n. 2, p. 107-116, 2005.

CARDOSO, V. D.; PALMA, L. E; ZANELLA, A. K. A motivação de pessoas com deficiência para a prática do esporte adaptado. Lecturas: Educación Física y Deportes, Revista Digital, Buenos Aires, v. 15, n. 146, 2010. Disponível em: <https://www.efdeportes.com/efd146/a-motivacao-para-esporte-adaptado.htm>. Acessado em: 29 de julho de 2019.

CESCHINI, F. L.; FIGUEIRA JUNIOR, A. Barreiras e determinantes para a prática de atividade física em adolescentes. Revista Brasileira de Ciência e Movimento, Brasília, v. 15, n. 1, p. 29-36, 2008

CHIAVENATO, I. Comportamento organizacional: a dinâmica do sucesso das organizações. Barueri: Manole, 2005.

CRATTY, B. J. Psicologia no esporte. Rio de Janeiro: Prentice-Hall, 1984.

FERRAZ, M. A. A preferência pela prática de atividades físicas e esportivas: uma abordagem psicofísica. 2005. 198 f. Tese (Doutorado em Ciências) - Universidade de São Paulo, São Paulo, 2005.

FRONTERA, W. R. Exercício físico e reabilitação. São Paulo: Artmed, 2001.

GIL, A. C. Como elaborar projeto de pesquisa. 4. ed. São Paulo: Atlas, 2002.

GREGUOL, M. Atividades físicas e esportivas e pessoas com deficiência. In: BOLZON, A.; GONZALEZ, J.; VELASCO, A.; OLIVEIRA, A. P. de; MANOEL, E. D. J.; IMPOLCETTO, F. M.; ...; DANTAS, L. E. P. B. T. Movimento é vida: atividades físicas e esportivas para todas as pessoas. Relatório De Desenvolvimento Humano do Brasil - PNUD. Brasília, 2017. p.16-375.

LOVISOLO, H. R. Estética, esporte e educação física. Rio de Janeiro: Sprint, 1997.

MACHADO, A. A. Psicologia do esporte: temas emergentes. Jundiaí: Ápice, 1997.

MARMELEIRA, J. F. F.; FERNANDES, J. M. G. A.; RIBEIRO, N. C.; TEIXEIRA, J. A.; GUTIERRES FILHO, P. J. B. Barreiras para a prática de atividade física em pessoas com deficiência visual. Revista Brasileira de Ciências do Esporte, Brasília, v. 40, n. 2, p. 197-204, 2018.

MARTINS, M. O.; PETROSKI, E. L. Mensuração da percepção de barreiras para a prática de atividades físicas: uma proposta de instrumento. Revista Brasileira Cineantropometria e Desempenho Humano, Florianópolis, v. 2, n. 1, p. 58-65, 2000.

MELO, A. C. R.; LÓPEZ, R. F. A. O Esporte Adaptado. Lecturas: Educación 
PALMA. L. E.; PATIAS, B. C.; FECK, R. M.

Física y Deportes, Revista Digital, Buenos Aires, v. 8, n. 51, 2002. Disponível em: <https://www.efdeportes.com/efd51/esporte.htm>. Acessado em: 06 de novembro de 2018 .

MINATTO, G.; GINAR, S.; FARES, D.; SANTOS, S. G. População e amostra/sujeitos da pesquisa. In: SANTOS, S. G (Org.). Métodos e técnicas de pesquisa quantitativa aplicada a educação física. Florianópolis: Tribo da Ilha, 2011. p. $129-140$

NAHAS, M. V. Atividade física, saúde e qualidade de vida: conceitos e sugestões para um estilo de vida ativo. Londrina: Midiograf, 2006.

NAHAS, M. V.; GARCIA, L. M. T. Um pouco de história, desenvolvimentos recentes e perspectivas para a pesquisa em atividade física e saúde no Brasil. Revista Brasileira de Educação Física e Esporte, São Paulo, v. 24, n. 1, p. 135-48, 2010.

PAGANI, L. G. Análise motivacional de atletas do paradesporto de nível nacional. Revista Brasileira de Prescrição e Fisiologia do Exercício, São Paulo, v. 6 , n. 34 , p. 330-5, 2012

PALMA, L.; FECK, R.; PATIAS, B.; LONDERO, A.; BAGATINI, G.; DORNELES, M.; MACHADO, V. Aspectos Motivacionais para a prática de Atividade Física por pessoas com deficiência visual. Kinesis, Santa Maria, v. 35, n. 2, p. 43-9, 2017.

PALMA, L. E.; FECK, R. M.; PATIAS, B. C.; FRIEDRICH, F. Tendências competitivas de atletas de basquetebol em cadeira de rodas. Caderno de Educação Física e Esporte, Marechal Cândido Rondon, v. 14, n. 1, p. 51-7, 2016.

SAITO, S. P. D. Motivos que levam indivíduos portadores de deficiência visual a participarem de atividades desportivas adaptadas de alto nível. Revista Mineira de Educação Física, Viçosa, v. 15, n. 2, p. 20-32, 2007

SAMPIERI, R. H.; COLLADO, C. F.; LUCIO, M. P. B. Metodologia de pesquisa. São Paulo: McGraw-Hill, 2006.

SAMULSKI, D. Psicologia do esporte: um manual para educação física, fisioterapia e psicologia. São Paulo: Manole, 2002.

SANTOS, S. C.; KNIJNIK, J. D. Motivos de adesão à prática de atividade física na vida adulta intermediária. Revista Mackenzie de Educação Física e Esporte, São Paulo, v. 5, n. 1, p. 23-34, 2009.

SARRAZIN, P. G.; BOICHÉ, J. C. S.; PELLETIER, L. G. A self-determination approach to sport dropout. In: HAGGER, M.; CHATZISATANTIS, N. (Eds.). Intrinsic motivation and self-determination in exercise and sport. Champaign: Human Kinetics, 2007.

SERON, B. B.; ARRUDA, G. A. de; GREGUOL, M. Facilitadores e barreiras percebidas para a prática de atividade física por pessoas com deficiência motora. Revista Brasileira de Ciências do Esporte, Brasília, v. 37, n. 3, p. 214-21, 2015.

SILVA, F. F.; SILVA, J. A. da; DUIM, G. A. S.; NASCIMENTO JUNIOR, J. R. A.; ARAÚJO, P. F. de. Orientação motivacional de atletas usuários de cadeira de rodas. Caderno de Educação Física e Esporte, Marechal Cândido Rondon, v. 17, n. 2, p. 13-19, 2019

SOUZA, M. M. de; AIRES, H.; GONÇALVES, G. H. T.; BALBINOTTI, C. A. A. MuIheres no futsal: motivos que levam à prática. Kinesis, Rio Claro, v. 35, n. 3, p. 101-8, 2017

THOMAS, J.R.; NELSON, J. K.; SILVERMAN, S. J. Métodos de pesquisa em atividade física. Porto Alegre: Artmed Editora, 2009.

TORRALBA, M. A.; VIEIRA, M. B.; E RUBIO, M. J. Motivos de La prácticadeportiva de atletas paralímpicos españoles. Revista de Psicología Del Deporte, Cerdanyola del Vallès, v. 26, n. 1, p. 49-60, 2017.

VASUDEVAN, V.; RIMMER, J. H.; KVIZ, F. Development of the barriers to physical activity questionnaire for people with mobility impairments. Disability and Health Journal, Amsterdam, v. 8, p. 547-56, 2015.

WHO. World Health Organization. International Classification of Functioning, Disability and Health (ICF). The World Bank, 2016. Disponível em: <http:// www.who.int/classifications/icf/en/>. Acesso em: 14 de junho de 2020.

\section{AGRADECIMENTOS}

Os autores agradecem a UFSM que nos proporcionou desenvolver esse estudo e a todos que participaram.

\section{CONFLITO DE INTERESSE}

Os autores do estudo declaram não haver conflito de interesses.

\section{FINANCIAMENTO}

Este estudo não teve apoio financeiro.

\section{ORCID E E-MAIL DOS AUTORES}

Luciana Erina Palma

ORCID: 0000-0003-2036-078.

E-mail: luepalma@yahoo.com.br

Bhianca Conterato Patias (Autor Correspondente)

ORCID: 0000-0002-0221-186X.

E-mail: bhiiancacp@gmail.com

Roberta Marostega Feck

ORCID: 0000-0002-1062-8585.

E-mail: nina.marostega@gmail.com 